Article

\title{
Histopathological Evaluation of Tumor-Infiltrating Lymphocytes (TILs) as Predictive Biomarker for Hormone Receptors Status, Proliferative Activity and Clinical Outcome in Her-2 Positive Breast Cancer
}

\author{
Giuseppe Angelico ${ }^{1,+}\left(\mathbb{D}\right.$, Giuseppe Broggi ${ }^{2,+}\left(\mathbb{D}\right.$, Rosario Caltabiano ${ }^{2}\left(\mathbb{D}\right.$, Angela Santoro $^{1}(\mathbb{D}$, \\ Saveria Spadola ${ }^{1}{ }^{10}$, Nicoletta D'Alessandris ${ }^{1}$, Giulia Scaglione ${ }^{1}$, Michele Valente ${ }^{1}$, Damiano Arciuolo ${ }^{1}$, \\ Alejandro Martin Sanchez ${ }^{3}(\mathbb{D})$, Gianluca Franceschini ${ }^{3,4}{ }^{\mathbb{D}}$, Riccardo Masetti ${ }^{3,4}$, Antonino Mulè 1 \\ and Gian Franco Zannoni ${ }^{1,5, *(D)}$
}

Citation: Angelico, G.; Broggi, G.; Caltabiano, R.; Santoro, A.; Spadola, S.; D'Alessandris, N.; Scaglione, G.; Valente, M.; Arciuolo, D.; Sanchez, A.M.; et al. Histopathological Evaluation of Tumor-Infiltrating Lymphocytes (TILs) as Predictive Biomarker for Hormone Receptors Status, Proliferative Activity and Clinical Outcome in Her-2 Positive Breast Cancer. Appl. Sci. 2021, 11, 6788. https://doi.org/ 10.3390/app11156788

Academic Editor: Laura Porretti

Received: 9 June 2021

Accepted: 16 July 2021

Published: 23 July 2021

Publisher's Note: MDPI stays neutral with regard to jurisdictional claims in published maps and institutional affiliations.

Copyright: (c) 2021 by the authors. Licensee MDPI, Basel, Switzerland. This article is an open access article distributed under the terms and conditions of the Creative Commons Attribution (CC BY) license (https:/ / creativecommons.org/licenses/by/ $4.0 /)$.
1 Unità di Gineco-Patologia e Patologia Mammaria, Dipartimento Scienze della Salute della Donna, del Bambino e di Sanità Pubblica, Fondazione Policlinico Universitario A. Gemelli IRCCS, L.go Francesco Vito 1, 00168 Rome, Italy; giuangel86@hotmail.it (G.A.); angelasantoro1@hotmail.it (A.S.); saveriaspadola@hotmail.it (S.S.); ndalessandris@gmail.com (N.D.); scaglione.giulia90@gmail.com (G.S.); dr.valente.m@gmail.com (M.V.); damiano.arciuolo@policlinicogemelli.it (D.A.); antonino.mule@policlinicogemelli.it (A.M.)

2 Department G.F. Ingrassia, Section of Pathology, University of Catania, 95123 Catania, Italy; giuseppe.broggi@gmail.com (G.B.); rosario.caltabiano@unict.it (R.C.)

3 Multidisciplinary Breast Center, Dipartimento Scienze della Salute della donna e del Bambino e di Sanità Pubblica, Fondazione Policlinico Universitario A. Gemelli IRCCS, Largo Agostino Gemelli 8, 00168 Rome, Italy; martin.sanchez@policlinicogemelli.it (A.M.S.); franceschinigianluca@gmail.com (G.F.); riccardo.masetti@policlinicogemelli.it (R.M.)

4 Istituto di Semeiotica Chirurgica, Università Cattolica del Sacro Cuore, 00168 Rome, Italy

5 Istituto di Anatomia Patologica, Università Cattolica del Sacro Cuore, 00168 Rome, Italy

* Correspondence: gianfranco.zannoni@unicatt.it

+ These authors have equally contributed.

Abstract: Background: In the present study, we evaluated the prognostic value of TILs as well their relation with clinicopathological factors in patients affected by HER-2 positive breast cancer. Methods: We evaluated 47 patients with a histologically confirmed diagnosis of invasive breast carcinoma showing an immunohistochemically confirmed (score 3+) amplification of the c-erbB-2 gene for the presence of TILs and categorized in three predefined groups of low (0-10\% immune cells in stromal tissue within the tumor), intermediate (11-40\%), and high TILs (>40\%). Results: Low, intermediate and high TILs were found in 17/47 (36\%), 23/47 (49\%) and 7/47(15\%) cases, respectively. It was found that $6 / 47$ cases treated with adjuvant chemotherapy plus trastuzumab underwent progression of the disease; none of these cases exhibited high TILs. It was found that $12 / 47$ patients with a prognostically unfavorable stage of III and IV showed low and intermediate levels of TILs, while high TILs were never observed. A significant association between intermediate/high-levels of TILs, elevated KI 67 index and hormone receptors nuclear staining was observed. High concordance in TILs distribution was observed between preoperative breast biopsies and surgical samples. Conclusions: We observed a positive correlation between the TILs and the response to both adjuvant and neoadjuvant treatments in HER-2 positive BC. High TILs were also related to increased KI-67 index and to the expression of hormone receptors.

Keywords: breast cancer; HER2; tumor infiltrating lymphocytes; KI-67; hormone receptors; immune microenvironment; target therapy

\section{Introduction}

Breast cancer $(B C)$ is the most frequent malignancy in women [1-3]; it exhibits a wide geographic variability, with higher incidence rates in the most economically advanced 
countries [1-3]. With respect to age groups, the incidence of BC tends to increase up to the menopausal years (around 50-55 years), slow down and then start to rise again after the age of 60 [2]. BC does not represent a homogeneous disease, but a wide spectrum of neoplasms characterized by huge differences in terms of clinical behavior, prognosis and response to treatments [1-3]. In recent years several studies have been carried out in order to identify a series of morphological or molecular parameters that were related to the patient's prognosis (i.e., the clinical course of the disease and the overall survival) or to the response to specific treatments (predictive factors) [1]. Some of these fall into both categories, such as the expression of estrogen receptors (ERs), HER-2, the proliferation index and genetic signatures [4].

The immunogenicity of BC has not traditionally been taken into account either in clinical practice or in scientific research, unlike other types of malignancies, such as melanoma, lung cancer and gynecological neoplasms, for which new therapies, able to stimulate the antitumor immune response have recently been introduced in clinical practice with a significant impact on prognosis [5-8]. However, in recent years numerous clinical studies have been conducted in order to better understand whether the individual immune response was able to influence the clinical behavior of $\mathrm{BC}$ and, in other words, whether it was possible to assign to each mammary neoplasms an "immunological grade" that had prognostic and/or predictive significance of therapeutic response [9]. The presence of tumor-infiltrating lymphocytes (TILs), within the tumor and/or in peritumoral sites, has been recognized as an important immunological biomarker that reflected the antitumor immune response in BC, as well as in other malignancies, including ovarian and endometrial carcinomas [9-11].

Several studies have attempted to determine the prognostic value of TILs in breast cancer. In detail, increased TIL levels have been linked to better response to neoadjuvant chemotherapy and with improved survival for patients with triple negative tumors (TNBC) and HER2-positive BC [12]. On the other hand, the prognostic and predictive value of TILs in luminal breast cancer remains poorly understood [12].

TILs are most often found in triple-negative breast cancers, HER2+, and other highly proliferative subtypes $[9,13]$. Some authors have extensively studied the composition of TILs: $75 \%$ of each TIL is made up of T lymphocytes, less than $20 \%$ of B lymphocytes, less than $10 \%$ of monocytes and less than $5 \%$ of natural killer (NK) and NK- T cells $[14,15]$. TILs are classified into stromal (sTILs) and intratumoral (iTILs) TILs, both located within the tumor tissue; however, while sTILs are composed of lymphocytes dispersed in the tumor stroma, up to and including the invasive front of the tumor, iTILs are made up of lymphocytes properly located within the tumor nests and therefore in direct contact with neoplastic cells $[9,14,15]$. sTILs represent a more reproducible parameter because they are more frequently encountered and therefore easier to detect in hematoxylin and eosin (H\&E)stained sections with no use of immunohistochemical methods [9,14-16]; to date, there is no standardized method for evaluating TILs in daily histopathological practice. In 2010 a method of evaluating TILs on needle biopsies samples was proposed by Denkert et al. [16] and, since then, it has been widely accepted. In 2014 an International TILs Working Group introduced a set of recommendations for a standardized evaluation of TILs in BC [14]. Based on the abovementioned acquisitions, the aim of the present study was to evaluate the potential prognostic and predictive role of TILs in patients affected by HER-2 positive BC. Since this subset of tumors is eligible to be treated with trastuzumab, a humanized anti-Human Epidermal growth factor Receptor 2 (HER2) receptor monoclonal antibody, we also aimed to evaluate a possible correlation between TILs levels and treatment response. Associations between TILs distributions and clinicopathological factors such as hormone receptors status and $\mathrm{Ki}-67$ proliferative index have also been investigated. 


\section{Materials and Methods}

\subsection{Patients Selection}

We retrospectively collected information about 47 patients, aged between 40 and 70 years, with a histologically confirmed diagnosis of invasive breast carcinomas showing an immunohistochemically confirmed (score $3+$ ) amplification of the c-erbB-2 gene from the files of our institution. Preoperative breast core needle biopsies (CNB) were also available for all the patients. All patients were treated with adjuvant chemotherapy plus trastuzumab, in particular 6/47 women (mean age: 69.5 years; age range: 53-82 years) were treated also with neo-adjuvant chemotherapy plus trastuzumab. All cases underwent a 2-3-year follow-up.

Expressions of estrogen (ER), progesterone (PR), HER2, and Ki-67 were assessed using immunohistochemistry, as elsewhere reported [4]. HER2-negative tumors were excluded from this study. Tumor proliferative activity was assessed by immunohistochemistry, using the Ki-67 antibody. In detail, at least three high power fields (HPFs) were selected to represent the spectrum of staining seen on the initial overview of the entire section. The Ki67 index was expressed as the percentage of positively stained cells among the total number of invasive cancer cells in the area scored. Scoring involved the counting of at least 500 malignant invasive cells with a $40 \times$ object lens.

All slides were prepared by standard techniques and scanned with Aperio ScanScope at $\times 20$. Digital images viewed in ImageScope (Aperio) image viewer which was also utilized to take photomicrographs.

\subsection{Histological Evaluation of TILs}

The evaluation of the TILs was performed according to the guidelines of the "International Working Group for TILs in Breast Cancer-2014" [14]. In detail, a section of 4-5 $\mu \mathrm{m}$ at a magnification of 200-400 $\times$ was evaluated for each patient. The evaluation of the TILs was performed by a percentage count of the stromal areas occupied by the lymphocyte and plasma cellular infiltrate, instead excluding the areas occupied by tumor cells. This evaluation considered only the mononuclear infiltrate within the borders of the invasive tumors. Large areas of central necrosis or fibrosis are not included in the evaluation.

The findings were categorized in three predefined groups of low $(0-10 \%$ immune cells in stromal tissue within the tumor), intermediate (11-40\%), and high TILs ( $>40 \%)$ as described by the International Working Group for TILs in Breast Cancer 2014 (14). For the intermediate group different areas at higher magnification were also considered.

TILs levels were also evaluated separately for core needle biopsies (CNBs) and surgical samples (SRS) and recorded as CNB-TILs and SRS-TILs. Tumors were categorized in the following subgroups: CNB-High-TILs, CNB-Intermediate-TILs, CNB-Low-TILs, SRS-High-TILs, SRS-Intermediate-TILs and SRS-Low-TILs, according to CNB-TILs and SRS-TILs status.

\subsection{Statistical Analysis}

Descriptive data were expressed as absolute values, relative percentages and mean or median with standard deviations. The agreement rate in TILs evaluation between CNBs and surgical samples was performed using Cohen's Kappa test $(\mathrm{k})$. The interpretation of the agreement by Kappa value was performed with the intervals: $k<0$, less than chance agreement; $\mathrm{k}=0.01-0.20$, slight agreement; $\mathrm{k}=0.21-0.40$, fair agreement; $\mathrm{k}=0.41-0.60$, moderate agreement; $\mathrm{k}=0.61-0.80$, substantial agreement; and $\mathrm{k}=0.81-0.99$, almost perfect agreement. $p$-values less than 0.05 were considered significant. To assess the correlations between TILs and clinicopathological variables, Fisher's exact test was performed using the SPPS Statistics 23 software (Statistical Package for Social Science, SPSS Inc, Armonk, New York, NY, USA). Statistical significance was defined when $p<0.05$. 


\section{Results}

\subsection{Patient's Characteristics and TILs Distribution}

The clinical-pathological features of the cohort of patients included in the study are summarized in Table 1.

Table 1. Patients' Characteristics.

\begin{tabular}{|c|c|}
\hline Total number of patients enrolled & 47 \\
\hline Median age at diagnosis (y) & 61 \\
\hline \multicolumn{2}{|l|}{ Tumor stage } \\
\hline I & $27(57.45 \%)$ \\
\hline II & $8(17 \%)$ \\
\hline III & $3(6.38 \%)$ \\
\hline IV & $9(19.15 \%)$ \\
\hline \multicolumn{2}{|l|}{ Histological type } \\
\hline Ductal NOS & $42(89.36 \%)$ \\
\hline Lobular & $4(8.51 \%)$ \\
\hline Metaplastic & $1(2.13 \%)$ \\
\hline \multicolumn{2}{|l|}{ Histological grade } \\
\hline $\mathrm{G} 2$ & $11(23.4 \%)$ \\
\hline G3 & $36(76.6 \%)$ \\
\hline \multicolumn{2}{|l|}{ Lymph node status } \\
\hline Negative & $28(59.57 \%)$ \\
\hline Micrometastases & $3(6.38 \%)$ \\
\hline Macrometastases & $16(34.04 \%)$ \\
\hline \multicolumn{2}{|c|}{ TILs (evaluation on CNB/surgical samples) } \\
\hline Low & $19 / 17$ \\
\hline Intermediate & $21 / 23$ \\
\hline High & $7 / 7$ \\
\hline \multicolumn{2}{|l|}{ Hormone receptors (ER, PR) } \\
\hline positive & $28(59.57 \%)$ \\
\hline negative & $19(40.43 \%)$ \\
\hline \multicolumn{2}{|l|}{ Ki-67 } \\
\hline$>20 \%$ & $36(76.6 \%)$ \\
\hline$\leq 20 \%$ & $11(23.4 \%)$ \\
\hline \multicolumn{2}{|l|}{ Chemotherapy } \\
\hline Only Adjuvant & $47(100 \%)$ \\
\hline Neoadjuvant+Adjuvant & $6(12.77 \%)$ \\
\hline \multicolumn{2}{|l|}{ Relapse } \\
\hline Yes & $6(12.77 \%)$ \\
\hline No & $41(87.23 \%)$ \\
\hline \multicolumn{2}{|l|}{ Surgical approach } \\
\hline Quadrantectomy & $43(93.49 \%)$ \\
\hline Mastectomy & $4(8.51 \%)$ \\
\hline \multicolumn{2}{|l|}{ Margin status } \\
\hline Free & 47 \\
\hline Ink on Tumor & 0 \\
\hline
\end{tabular}

After evaluating the percentage of TILs, all the cases of HER-2 positive breast carcinomas from our cohort were assigned to groups $\mathrm{A}, \mathrm{B}$ and $\mathrm{C}$, corresponding to the groups of low $(0-10 \%)$, intermediate $(10-40 \%)$ and high (40-90\%) TILs, respectively, as described by the International Working Group for TILs in Breast Cancer 2014 (14). Low, intermediate and high TILs were found in 17/47 (36\%), 23/47 (49\%) and 7/47(15\%) patients, respectively (Figures 1 and 2) (evaluation of surgical samples). Considering TILs assessment on CNBs from the same patients, low, intermediate and high TILs were found in 19/47 (40.4\%), $21 / 47(44.6 \%)$ and $7 / 47(15 \%)$ cases, respectively. 


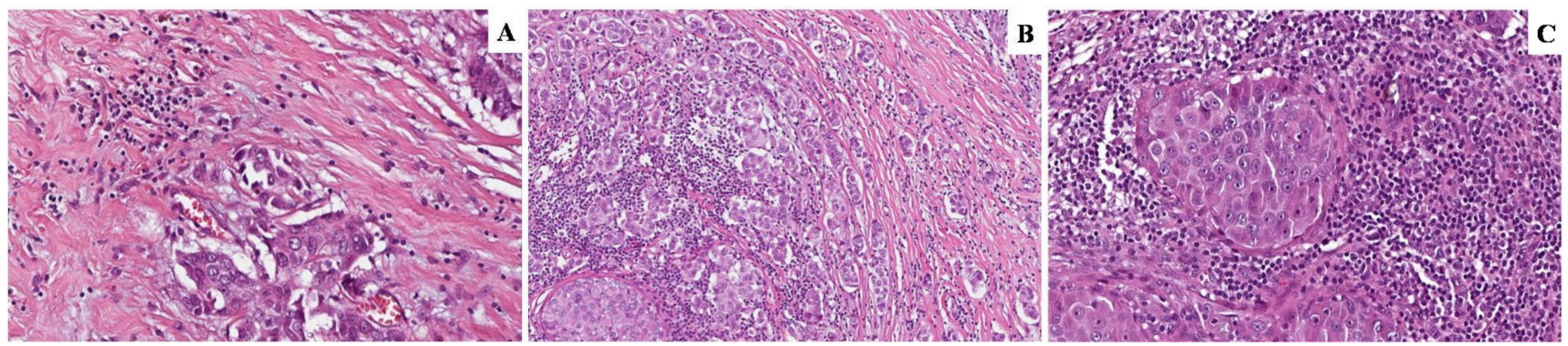

Figure 1. Hematoxylin and eosin (H\&E) stained sections illustrating different TILs distribution in our series. (A) Invasive breast carcinoma with low TILs (0-10\%) in stromal tissue within the tumor $(\mathrm{H} \& \mathrm{E}, \times 10)$. (B) Another case in our series demonstrating increased TILs in tumor stroma, categorized as intermediate $(11-40 \%)(\mathrm{H} \& \mathrm{E}, \times 10)$. (C) High TILs (>40\%) in tumor stroma are depicted in this invasive breast cancer case $(\mathrm{H} \& \mathrm{E}, \times 20)$.
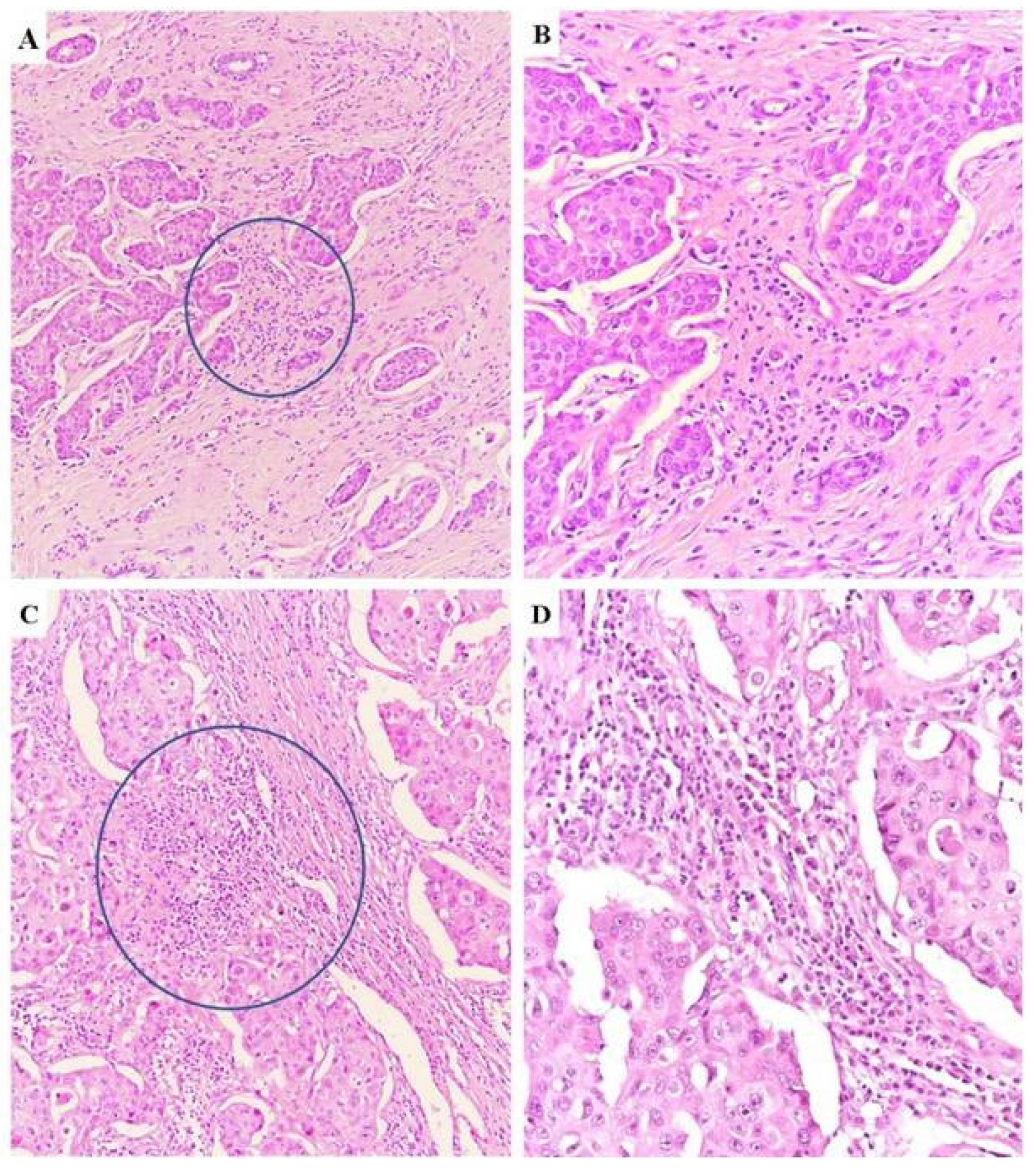

Figure 2. Hematoxylin and eosin (H\&E) stained sections illustrating different TILs distribution in our series. (A) Invasive breast carcinoma demonstrating low TILs $(H \& E, \times 10)$. (B) Higher magnification of the same case $(\mathrm{H \& E}, \times 20)$ illustrating the TILs distribution in tumor stroma. $(\mathbf{C})$ Another invasive breast cancer case categorized as intermediate TILs $(H \& E, \times 10)$. (D) Higher magnification of the same case $(\mathrm{H} \& \mathrm{E}, \times 20)$ demonstrating the increased TILs distribution in tumor stroma.

\subsection{TILs and Clinicopathological Features}

Of the 47 cases of HER-2 positive BC considered here, six were treated with treated with adjuvant chemotherapy plus trastuzumab but underwent progression of disease (local recurrence): the average time to progression for these patients was 10 months; five of the six cases that progressed showed low TILs $(0-10 \%)$ at the time of the histological diagnosis, in addition to an absent or mild peritumoral lymphocytic infiltrate; the remaining relapsed case TILs levels were intermediate, equal to about $25 \%$, with a moderate peritumoral 
lymphocytic infiltrate; notably, none of the 6 relapsed cases exhibited high TILs (40-90\%). Considering the surgical management of these patients, two patients underwent mastectomy, while in four patients a more conservative approach was preferred (quadrantectomy). In all patients, clear margins were reported.

Taking into consideration tumor stage, according to The American Joint Committee on Cancer (AJCC) staging, 12/47 patients showed a prognostically unfavorable stage of III and IV (Table 1); this subgroup of patients showed low levels of TILs, while intermediate and high TILs were never observed (Table 2). No other significant correlation has emerged between TILs distribution and clinicopathological variables.

Table 2. Relation between TILs and stage.

\begin{tabular}{cccc}
\hline & Stage III-IV & Stage I-II & Total \\
\hline LOW TILs & $12(25.53 \%)$ & $15(31.91 \%)$ & 27 \\
\hline I-HIGH TILs & 0 & $20(42.55 \%)$ & 20 \\
\hline Total & 12 & 35 & 47 \\
\hline$p$-value & & 0.0004 & \\
\hline
\end{tabular}

Considering nodal status, 19/47 patients showed metastatic nodes (Table 1); this subgroup of patients showed low levels of TILs in 12 cases, while intermediate-high TILs were observed in 7 cases (Table 3 ). A positive nodal status was significantly related to low TILs ( $p$-value: 0.006 )

Table 3. Relation between TILs and nodal status.

\begin{tabular}{cccc}
\hline & $\mathbf{N}+$ & $\mathbf{N}-$ & Total \\
\hline LOW TILs & $12(25.53 \%)$ & $6(12.77 \%)$ & 27 \\
\hline I-HIGH TILs & $7(14.89 \%)$ & $22(46.81 \%)$ & 20 \\
\hline Total & 19 & 28 & 47 \\
\hline$p$-value & & 0.006 &
\end{tabular}

In regard to the correlation between TILs and tumor response to neoadjuvant chemotherapy, our series included only six cases of locally advanced HER-2 positive BC, preoperatively treated with a combination of anthracyclines, taxanes and trastuzumab. All cases examined showed low TILs and none of them underwent tumor pathological complete response (pCR). In addition, $1 / 6$ cases exhibited local disease progression. From an immunohistochemical point of view, in two patients, the neoplasms were luminal HER2 (HR+/HER2+) BCs; in four patients, the neoplasms were pure HER2 (HR-/HER2+, one of which also exhibited immunopositivity for basal CKs-CK14, CK5/6 and focally for CK17, as a basal HER2: HR-/HER2+/basal CKs+).

No statistically significant associations have been observed between TIL status, histotype and pathological grade.

\subsection{Relation between TILs, Hormone Receptors and Proliferative Index (Ki-67)}

We assessed whether the proportion of TILs was associated with estrogen and progesterone staining patterns as well as Ki-67. Interestingly, a statistically significant association between intermediate and high-levels of TILs and a Ki-67 index $<20 \%$ was observed ( $p$-value: 0.0094) (Table 4). 
Table 4. Relation between TILs and proliferative index (Ki-67).

\begin{tabular}{cccc}
\hline & Ki-67 $>\mathbf{2 0} \%$ & Ki-67 $\leq \mathbf{2 0} \%$ & Total \\
\hline LOW TILs & $9(19.15 \%)$ & $8(17.02 \%)$ & 17 \\
\hline I-HIGH TILs & $27(57.45 \%)$ & $3(6.38 \%)$ & 30 \\
\hline Total & 36 & 11 & 47 \\
\hline$p$-value & & 0.0094 & \\
\hline
\end{tabular}

Moreover, the nuclear staining of estrogen and progesterone receptors, observed in 28/47 cases, was significantly related to intermediate-high TILs ( $p$-value: 0.0150) (Table 5).

Table 5. Relation between TILs and hormone receptors.

\begin{tabular}{cccc}
\hline TILs & LOW & $\begin{array}{c}\text { INTERMEDIATE- } \\
\text { HIGH }\end{array}$ & Total \\
\hline HR+/HER2+ & $6(12.77 \%)$ & $22(46.81 \%)$ & 28 \\
\hline HR-/HER2+ & $11(23.4 \%)$ & $8(17.02 \%)$ & 19 \\
\hline Total & 17 & 30 & 47 \\
\hline$p$-value & & 0.0150 & \\
\hline
\end{tabular}

\subsection{Concordance of TILs Evaluation between CNB and Surgical Samples}

On the basis of the number of observed agreements ( $100 \%$ of the observations) and the number of agreements expected by chance $(53.83,65 \%$ of the observations), the statistical analysis of specific Cohen's kappa values showed almost perfect agreement between CNB and surgical samples in the intermediate and high TILs groups $(\mathrm{k}=1000,95 \%$ confidence interval from 1000 to 1.000). On the other hand, two cases classified as low TILs in the CNB evaluation revealed intermediate TILs in breast surgical samples $(\mathrm{k}=0.905,95 \% \mathrm{CI}$, from 0.778 to 1000). In detail, all tumors with high TILs on CNBs were confirmed on surgical samples. On the other hand, 2/19 tumors categorized as low TILS on CNB evaluation were upgraded to intermediate TILs on surgical sample examination. This difference is explained by the heterogeneity of TILs' distribution on definitive examination of these tumors (Table 6).

Table 6. TILs distribution in CNB vs. surgical samples.

\begin{tabular}{cccc}
\hline & \multicolumn{3}{c}{ TILs } \\
\hline LOW & INTERMEDIATE & HIGH \\
\hline $\begin{array}{c}\text { CNB } \\
\text { SAMPICAL }\end{array}$ & 19 & 21 & 7 \\
\hline
\end{tabular}

\section{Discussion}

In recent years, the immunogenicity of breast cancer has become subject of great scientific attention, with the aim of better understanding whether it can influence the biological behavior of $\mathrm{BC}$, acquiring prognostic and/or predictive significance of therapeutic response $[9,12,14-16]$. The presence of TILs is an expression of the antitumor immune response. Many studies have therefore focused on the predictive and/or prognostic impact of TILs in patients with different types of cancers, including BC [9-11]. TILs are classified into stromal and intratumoral TILs, depending on whether we consider the lymphocytes dispersed in the tumor stroma, or the lymphocytes located within the tumor nests $[9,14,15]$. Due to the absence of a standardized method for evaluating TILs, many studies have evaluated both the sTILs and the iTILs; however, the evaluation of the former was easier to 
detect and more reproducible [9,14-16]. In 2014 a series of recommendations for a standardized assessment of TILs in breast cancer was proposed by an international TILs working group [14]; in addition, several studies demonstrated the prognostic and predictive value of TILs in various cohorts of patients; particularly, among triple negative BCs (TNBCs), TILs can be used as a prognostic marker because they positively correlated with the response to adjuvant chemotherapy, in terms of overall survival [9,12,16-18]. Recent studies aimed to investigate the potential prognostic role of TILs in patients affected by HER-2 positive BC treated with chemotherapy plus trastuzumab. In this regard, the FIN-HER study [18] demonstrated that patients affected by BC exhibiting high TILs levels showed a better response to adjuvant chemotherapy with trastuzumab and, consequently, longer overall survival than those affected by BC showing low TILs. On the other hand, Perez et al. [19] reported that HER2-positive lymphocytic-dominant $\mathrm{BCs}$, defined by the presence of at least $60 \%$ sTILs, had a slightly worse response when combining trastuzumab with chemotherapy than those treated with chemotherapy alone in terms of relapse-free survival times $(80 \%$ and $90.9 \%$ at 10 years, respectively). Therefore, based on these data, the potential role of TILs in mediating the response to adjuvant chemotherapy plus trastuzumab still remains controversial. Further data from neoadjuvant therapy-based studies $[20,21]$ showed that high rates of TILs were significantly associated with higher rates of pCR; accordingly, TILs appeared as predictive biomarkers of positive response/resistance to neoadjuvant chemotherapy. Post-neoadjuvant treatment TILs have also been found to be associated with breast cancer prognosis [22,23].

The aim of the present study was to demonstrate that the evaluation of TILs was capable of identifying, among 47 patients with HER-2 positive BC, specific subgroups of patients characterized by different prognostic and clinical features. In this regard, we found that only the patients (6/47) who underwent disease progression after adjuvant chemotherapy plus trastuzumab had TILs $<25 \%$. Moreover, neoadjuvant treatment failed to achieve a pathological complete response in all BC cases from our series (6/47); interestingly these latter cases exhibited low TILs.

Previous studies have documented high TILs levels in aggressive breast cancer subtypes, and showed a possible relation between high TILs, Ki-67, ER and PR immunohistochemical expression $[9,24,25]$.

Similarly, in the present study, we observed a correlation between TILs distribution, proliferative index (Ki-67) and hormone receptors. In detail, intermediate and high-levels of TILs were significantly related to a Ki-67 index $<20 \%$. We selected a $20 \% \mathrm{Ki}-67$ cutoff according to previous studies by other authors on TILs in HER-2 positive BC [19,23]; moreover, a Ki- 67 index greater than $20 \%$ is generally considered the watershed between luminal A and luminal B tumors [3,4].

Regarding hormone receptors' expression, intermediate-high TILs were significantly related to $\mathrm{BC}$ cases showing nuclear staining for both estrogen and progesterone receptors. Finally, there are limited data regarding the concordance or the variability of immune profiles between CNBs and whole tumor sections from surgical procedures [26]. Some authors have reported immune-activation after CNB, with TILs being higher in SRS than in CNB [26]; other studies performed on mouse models suggested a biopsy-mediated development of an increasingly immunosuppressive tumor microenvironment [27]. Different hypotheses have been postulated in order to explain the possible reasons of TILs changes: (1) the CNB process as primary cause of immune-activation; (2) the concept of intratumor heterogeneity; (3) younger age and longer surgery time interval as indicators of the higher chance of TILs' increase after CNB [26-28].

In our study, we observed a high level of concordance between preoperative biopsies and surgical samples in TILs' distribution. In detail, almost perfect agreement was observed in the intermediate and high TILs groups.

We can postulate that the reason for this high concordance rate could rely on the adequate bioptical sampling of the neoplastic tissue (at least four bioptic fragments from different points of the tumoral tissue, in almost the totality of our study cases). On the 
other hand, two cases were preoperatively classified as low TILs in the CNB evaluation but changed from a low immune status to an intermediate TILs status on surgical samples and experienced a worse breast cancer-free interval. In particular, in these non-concordant cases only 1-2 bioptic fragments were obtained in the preoperative setting. This TILs' change may be explained by: (1) the spatial heterogeneity of lymphocytic infiltrate or the regional segregation of immune cells in some tumors, which can be missed in bioptic cores; (2) the not completely adequate bioptical sampling; (3) a dysfunctional immune infiltrate. The bad prognosis observed in these two cases was quite different from the common view that TILs' increase is related to better survival (especially in TNBCs) but is similar to a recent study on HER2+ tumors in which TILs' heterogeneity was associated with poor prognosis [28].

\section{Conclusions}

In conclusion, the results from the present study demonstrate a positive correlation between TILs and the response to adjuvant treatments in HER-2 positive BC. Furthermore, we found that all the patients who presented an advanced stage of the disease at the time of the diagnosis showed low levels of TILs. Increased TILs concentration may also be indicative of a subset of tumors with elevated Ki-67 index and with the expression of estrogen and progesterone receptors, which tend to be more responsive to chemotherapy. Our data also support the hypothesis that a subset of HER2+ BC is immunogenic and may harbor a different biological and pathogenetic pathway which includes microsatellite instability, as also documented in tumors of the gynecological tract $[7,8,29]$. These latter neoplasms may also be treated with immune-modulating therapies.

We are aware of the limitations of our study: the sample size, the retrospective nature and the short follow-up period, which is not fully adequate to assess tumor biology.

Further studies on larger series and with longer more meaningful follow-up length are needed in order to elucidate the prognostic role of TILs as well as their utility as a predictive biomarker for the therapeutic management of $\mathrm{BC}$ patients.

Author Contributions: Conceptualization, G.A., R.C. and G.B.; methodology, A.S. and R.C.; software, S.S. and M.V.; validation, R.M., N.D. and G.S.; formal analysis, G.F.Z., G.F. and A.M.; investigation, A.M.; resources, G.F.Z.; data curation, G.B., D.A.; writing—original draft preparation, G.A.; writing—review and editing, G.B.; visualization, N.D. and M.V.; supervision, A.M.S. and A.M.; project administration, A.M.S. and G.F.Z. All authors have read and agreed to the published version of the manuscript.

Funding: This research received no external funding.

Institutional Review Board Statement: The study complied with the Ethical Principles for Medical Research Involving Human Subjects according to the World Medical Association Declaration of Helsinki. The clinical information had been retrieved from the patients' medical records and pathology reports. Patients' initials or other personal identifiers did not appear in any image. Finally, all samples were anonymized before histology and immunohistochemistry. Therefore, no further ethical approval was necessary to perform the study.

Informed Consent Statement: The non-interventional, retrospective nature of our study did not require any informed consent, even if written informed consent had been obtained from each patient before surgical procedures.

Data Availability Statement: All data are available after reasonable request to the corresponding author.

Conflicts of Interest: The authors declare no conflict of interest.

\section{References}

1. Broggi, G.; Filetti, V.; Ieni, A.; Rapisarda, V.; Ledda, C.; Vitale, E.; Varricchio, S.; Russo, D.; Lombardo, C.; Tuccari, G.; et al. MacroH2A1 Immunoexpression in Breast Cancer. Front. Oncol. 2020, 10. [CrossRef]

2. Cammarata, F.P.; Forte, G.I.; Petringa, G.; Cirrone, G.; Cuttone, G.; Acquaviva, R.; Caltabiano, R.; Russo, G.; Broggi, G.; Bravatà, V.; et al. Molecular Investigation on a Triple Negative Breast Cancer Xenograft Model Exposed to Proton Beams. Int. J. Mol. Sci. 2020, 21, 6337. [CrossRef] 
3. Magro, G.; Salvatorelli, L.; Puzzo, L.; Piombino, E.; Bartoloni, G.; Broggi, G.; Vecchio, G. Practical approach to diagnosis of bland-looking spindle cell lesions of the breast. Pathologica 2019, 111, 344-360. [CrossRef] [PubMed]

4. Adamo, B.; Ricciardi, G.R.R.; Ieni, A.; Franchina, T.; Fazzari, C.; Sanò, M.V. The prognostic significance of combined androgen receptor, E-Cadherin, Ki67 and CK5/6 expression in patients with triple negative breast cancer. Oncotarget 2017, 8, 76974-76986. [CrossRef]

5. Bersanelli, M.; Petrelli, F.; Buti, S.; Stanganelli, I. Immune checkpoint inhibitors in adjuvant setting after radical resection of melanoma: A meta-analysis of the pivotal trials. Hum. Vaccines Immunother. 2021, 21, 1-6. [CrossRef]

6. Wu, L.-G.-J.; Zhou, D.-N.; Wang, T.; Ma, J.-Z.; Sui, H.; Deng, W.-L. The efficacy and safety of PD-1/PD-L1 inhibitors versus chemotherapy in patients with previously treated advanced non-small-cell lung cancer. Medicine 2021, 100, e25145. [CrossRef] [PubMed]

7. Zhang, C.; Yang, Q. Predictive Values of Programmed Cell Death-Ligand 1 Expression for Prognosis, Clinicopathological Factors, and Response to Programmed Cell Death-1/Programmed Cell Death-Ligand 1 Inhibitors in Patients with Gynecological Cancers: A Meta-Analysis. Front. Oncol. 2021, 10. [CrossRef]

8. Santoro, A.; Angelico, G.; Inzani, F.; Spadola, S.; Arciuolo, D.; Valente, M.; Musarra, T.; Capelli, G.; Fanfani, F.; Gallotta, V.; et al. Pathological features, immunoprofile and mismatch repair protein expression status in uterine endometrioid carcinoma: Focus on MELF pattern of myoinvasion. Eur. J. Surg. Oncol. 2021, 47, 338-345. [CrossRef]

9. Denkert, C.; Von Minckwitz, G.; Darb-Esfahani, S.; Lederer, B.; Heppner, B.I.; Weber, K.E.; Budczies, J.; Huober, J.; Klauschen, F.; Furlanetto, J.; et al. Tumour-infiltrating lymphocytes and prognosis in different subtypes of breast cancer: A pooled analysis of 3771 patients treated with neoadjuvant therapy. Lancet Oncol. 2018, 19, 40-50. [CrossRef]

10. Tomšová, M.; Melichar, B.; Sedláková, I.; Šteiner, I. Prognostic significance of CD3+ tumor-infiltrating lymphocytes in ovarian carcinoma. Gynecol. Oncol. 2008, 108, 415-420. [CrossRef]

11. De Jong, R.A.; Leffers, N.; Boezen, H.M.; ten Hoor, K.A.; van der Zee, A.G.; Hollema, H.; Nijman, H.W. Presence of tumorinfiltrating lymphocytes is an independent prognostic factor in type I and II endometrial cancer. Gynecol. Oncol. 2009, 114, 105-110. [CrossRef] [PubMed]

12. Gao, Z.-H.; Li, C.-X.; Liu, M.; Jiang, J.-Y. Predictive and prognostic role of tumour-infiltrating lymphocytes in breast cancer patients with different molecular subtypes: A meta-analysis. BMC Cancer 2020, 20, 1150. [CrossRef]

13. Coates, A.S.; Winer, E.P.; Goldhirsch, A.; Gelber, R.D.; Gnant, M.; Piccart-Gebhart, M.; Thürlimann, B.; Senn, H.-J.; Members, P.; Andre, F.; et al. Tailoring therapies-Improving the management of early breast cancer: St Gallen International Expert Consensus on the Primary Therapy of Early Breast Cancer 2015. Ann. Oncol. 2015, 26, 1533-1546. [CrossRef] [PubMed]

14. Salgado, R.; Denkert, C.; Demaria, S.; Sirtaine, N.; Klauschen, F.; Pruneri, G.; Wienert, S.; Eynden, G.V.D.; Baehner, F.L.; PenaultLlorca, F.; et al. The evaluation of tumor-infiltrating lymphocytes (TILs) in breast cancer: Recommendations by an International TILs Working Group 2014. Ann. Oncol. 2015, 26, 259-271. [CrossRef]

15. Mahmoud, S.; Paish, E.C.; Powe, D.G.; Macmillan, R.D.; Grainge, M.; Lee, A.H.; Ellis, I.; Green, A. Tumor-Infiltrating CD8+ Lymphocytes Predict Clinical Outcome in Breast Cancer. J. Clin. Oncol. 2011, 29, 1949-1955. [CrossRef]

16. Denkert, C.; Loibl, S.; Noske, A.; Roller, M.; Müller, B.M.; Komor, M.; Budczies, J.; Darb-Esfahani, S.; Kronenwett, R.; Hanusch, C.; et al. Tumor-Associated Lymphocytes As an Independent Predictor of Response to Neoadjuvant Chemotherapy in Breast Cancer. J. Clin. Oncol. 2010, 28, 105-113. [CrossRef]

17. Adams, S.; Gray, R.J.; Demaria, S.; Goldstein, L.; Perez, E.A.; Shulman, L.N.; Martino, S.; Wang, M.; Jones, V.E.; Saphner, T.J.; et al. Prognostic Value of Tumor-Infiltrating Lymphocytes in Triple-Negative Breast Cancers from Two Phase III Randomized Adjuvant Breast Cancer Trials: ECOG 2197 and ECOG 1199. J. Clin. Oncol. 2014, 32, 2959-2966. [CrossRef]

18. Loi, S.; Michiels, S.; Salgado, R.; Sirtaine, N.; Jose, V.; Fumagalli, D.; Kellokumpu-Lehtinen, P.-L.; Bono, P.; Kataja, V.; Desmedt, C.; et al. Tumor infiltrating lymphocytes are prognostic in triple negative breast cancer and predictive for trastuzumab benefit in early breast cancer: Results from the FinHER trial. Ann. Oncol. 2014, 25, 1544-1550. [CrossRef]

19. Perez, E.A.; Ballman, K.V.; Tenner, K.S.; Thompson, E.A.; Badve, S.; Bailey, H.D.; Baehner, F.L. Association of Stromal TumorInfiltrating Lymphocytes with Recurrence-Free Survival in the N9831 Adjuvant Trial in Patients With Early-Stage HER2-Positive Breast Cancer. JAMA Oncol. 2016, 2, 56-64. [CrossRef]

20. Mao, Y.; Qu, Q.; Zhang, Y.; Liu, J.; Chen, X.; Shen, K. The Value of Tumor Infiltrating Lymphocytes (TILs) for Predicting Response to Neoadjuvant Chemotherapy in Breast Cancer: A Systematic Review and Meta-Analysis. PLoS ONE 2014, 9, e115103. [CrossRef]

21. Yamaguchi, R.; Tanaka, M.; Yano, A.; Tse, G.M.; Yamaguchi, M.; Koura, K.; Kanomata, N.; Kawaguchi, A.; Akiba, J.; Naito, Y.; et al. Tumor-infiltrating lymphocytes are important pathologic predictors for neoadjuvant chemotherapy in patients with breast cancer. Hum. Pathol. 2012, 43, 1688-1694. [CrossRef]

22. Dieci, M.V.; Criscitiello, C.; Goubar, A.; Viale, G.; Conte, P.; Guarneri, V.; Ficarra, G.; Mathieu, M.C.; Delaloge, S.; Curigliano, G.; et al. Prognostic value of tumor-infiltrating lymphocytes on residual disease after primary chemotherapy for triple-negative breast cancer: A retrospective multicenter study. Ann. Oncol. 2015, 26, 611-618. [CrossRef]

23. Hamy, A.-S.; Pierga, J.-Y.; Sabaila, A.; Laas, E.; Bonsang-Kitzis, H.; Laurent, C.; Vincent-Salomon, A.; Cottu, P.; Lerebours, F.; Rouzier, R.; et al. Stromal lymphocyte infiltration after neoadjuvant chemotherapy is associated with aggressive residual disease and lower disease-free survival in HER2-positive breast cancer. Ann. Oncol. 2017, 28, 2233-2240. [CrossRef] [PubMed] 
24. Miyoshi, Y.; Shien, T.; Ogiya, A.; Ishida, N.; Yamazaki, K.; Horii, R.; Horimoto, Y.; Masuda, N.; Yasojima, H.; Inao, T.; et al. Associations in tumor infiltrating lymphocytes between clinicopathological factors and clinical outcomes in estrogen receptorpositive/human epidermal growth factor receptor type 2 negative breast cancer. Oncol. Lett. 2018, 17, 2177-2186. [CrossRef]

25. Loi, S.; Sirtaine, N.; Piette, F.; Salgado, R.; Viale, G.; Van Eenoo, F.; Rouas, G.; Francis, P.; Crown, J.P.; Hitre, E.; et al. Prognostic and Predictive Value of Tumor-Infiltrating Lymphocytes in a Phase III Randomized Adjuvant Breast Cancer Trial in Node-Positive Breast Cancer Comparing the Addition of Docetaxel to Doxorubicin with Doxorubicin-Based Chemotherapy: BIG 02-98. J. Clin. Oncol. 2013, 31, 860-867. [CrossRef] [PubMed]

26. Huang, J.; Chen, X.; Fei, X.; Huang, O.; Wu, J.; Zhu, L.; He, J.; Chen, W.; Li, Y.; Shen, K. Changes of Tumor Infiltrating Lymphocytes after Core Needle Biopsy and the Prognostic Implications in Early Stage Breast Cancer: A Retrospective Study. Cancer Res. Treat. 2019, 51, 1336-1346. [CrossRef] [PubMed]

27. Jeselsohn, R.M.; Werner, L.; Regan, M.M.; Fatima, A.; Gilmore, L.; Collins, L.C.; Beck, A.H.; Bailey, S.T.; He, H.H.; Buchwalter, G.; et al. Digital Quantification of Gene Expression in Sequential Breast Cancer Biopsies Reveals Activation of an Immune Response. PLoS ONE 2013, 8, e64225. [CrossRef] [PubMed]

28. Khan, A.M.; Yuan, Y. Biopsy variability of lymphocytic infiltration in breast cancer subtypes and the ImmunoSkew score. Sci. Rep. 2016, 6, 36231. [CrossRef]

29. Zannoni, G.F.; Santoro, A.; Angelico, G.; Spadola, S.; Arciuolo, D.; Valente, M.; Inzani, F.; Pettinato, A.; Vatrano, S.; Fanfani, F.; et al. Clear cell carcinoma of the endometrium: An immunohistochemical and molecular analysis of 45 cases. Hum. Pathol. 2019, 92, 10-17. [CrossRef] 